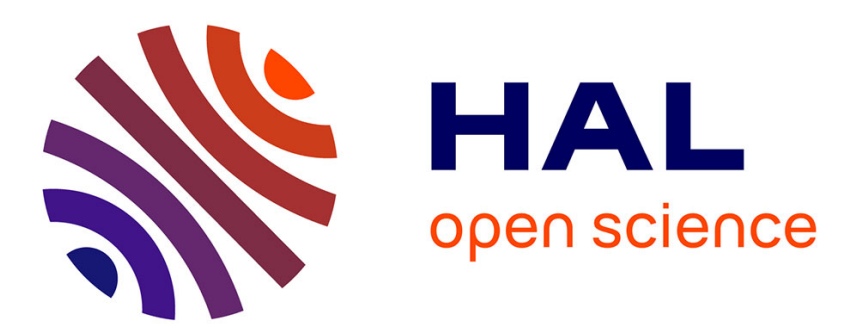

\title{
Strain profiles in thin films: influence of a coherently diffracting substrate and thickness fluctuations
}

\author{
Alexandre Boulle, F. Conchon, R. Guinebretière
}

\section{To cite this version:}

Alexandre Boulle, F. Conchon, R. Guinebretière. Strain profiles in thin films: influence of a coherently diffracting substrate and thickness fluctuations. Journal of Applied Crystallography, 2009, 42 (1), pp.85-92. 10.1107/S0021889808036406 . hal-02193848

\section{HAL Id: hal-02193848 \\ https://hal.science/hal-02193848}

Submitted on 24 Jul 2019

HAL is a multi-disciplinary open access archive for the deposit and dissemination of scientific research documents, whether they are published or not. The documents may come from teaching and research institutions in France or abroad, or from public or private research centers.
L'archive ouverte pluridisciplinaire HAL, est destinée au dépôt et à la diffusion de documents scientifiques de niveau recherche, publiés ou non, émanant des établissements d'enseignement et de recherche français ou étrangers, des laboratoires publics ou privés. 


\title{
Strain profiles in thin films:
}

\section{influence of a coherently diffracting substrate and thickness fluctuations}

\author{
A. Boulle, F. Conchon and R. Guinebretière \\ Laboratoire Science des Procédés Céramiques et de Traitements de Surfaces (SPCTS), CNRS \\ UMR 6638, ENSCI, 47 avenue Albert Thomas, 87065 Limoges - France
}

\begin{abstract}
A simple least-square fitting - based method is described for the determination of strain profiles in epitaxial films using high-resolution X-ray diffraction. The method is modelindependent, i.e. it does not require any "guess" model for the shape of the strain profile. The shape of the vertical displacement profile is modeled using the versatile cubic B-spline functions, which puts smoothness and curvature constraints on the fitting procedure. The effect of a coherently diffracting substrate is taken into account as well as the effects of film thickness fluctuations. The model is applied to the determination of strain profiles in $\mathrm{SmNiO}_{3}$ films epitaxially grown on (001) $\mathrm{SrTiO}_{3}$ substrates. The shape of the retrieved strain profile is discussed in terms of oxygen vacancies.
\end{abstract}

\section{Introduction}

Strain profiles, i.e. variations of the lattice parameter with depth, are frequently encountered in the processing of functional (e.g. semiconducting, optical, ferroelectric...) materials. In the last three decades much effort has been devoted to recover these strain profiles in a nondestructive way, especially using high-resolution X-ray diffraction (XRD). A well known 
example is the determination of strain profiles consecutive to ion implantation in semiconducting single crystals, as for instance Si (Diaz et al., 2007; Klappe \& Fewster, 1994; Milita \& Servidori, 1995; Sousbie et al., 2006; Zaumseil et al., 1987), SiC (Leclerc et al., 2005) or GaAs (Wierzchowski et al., 2005). Strain profiles also occur in epitaxial films as a result of the film substrate lattice mismatch and the associated strain relaxation (Nicola et al., 2005). Such strain profiles may profoundly affect the properties of the films, e.g. the ferroelectric (Catalan et al., 2005), optical (Siegle et al., 1997) or transport (Conchon et al., 2008a) properties.

The determination of strain profiles from experimental XRD data is hindered by the so-called "phase problem". Indeed, lattice displacements affect the phase of the diffracted amplitude, $E$, whereas the quantity measured experimentally is the intensity, E. $E^{*}$. The phase of the amplitude is hence lost so that the strain profile can not be obtained by inversion of the diffracted intensity (Nikulin, 1998; Vartanyants et al., 2000). This problem is usually solved using a model-dependent approach where a calculated XRD curve is least-square fitted to the experimental data. If the defect structure is known then the strain profile can be modeled by a physically sound model (Klappe \& Fewster, 1994; Milita \& Servidori, 1995; Hironaka et al., 2000). In these approaches the film is divided into laminae of constant strain so that the scattering problem can be solved dynamically within the framework of the Takagi (1969) Taupin (1964) equations (see also Bartels et al. (1986) and Halliwell et al. (1984)). Conversely, if the defect structure is unknown, i.e. there is no "guess" model for the strain profile, then any arbitrary function can be chosen (Shen \& Kycia, 1997; Steinfort et al., 1996). In both cases however, even if a good fit is achieved, if the chosen strain profile function does not match the actual shape of the strain profile, then the validity of the results is obviously questionable. More sophisticated, model-independent approaches, rely on the mathematical 
properties of the diffracted amplitude (in particular the fact that within the kinematical scattering theory, the diffracted amplitude is the Fourier transform of the electron density) to recover the phase of the amplitude using advanced "phase-retrieval" algorithms (Nikulin, 1998; Vartanyants et al., 2000; van der Veen \& Pfeiffer, 2004). The main advantages of these methods are that they don't depend on any a priori model, and that the obtained solution is in principle unambiguous. The drawback is that they rely on more complicated formalisms.

In a previous article (Boulle et al., 2003) we presented a method that combines the simplicity of least-square fitting based procedures with the advantage of being model-independent. In the present article we further develop this method. In particular we include the effects of the dynamical diffraction from the substrate together with thickness fluctuations of the film $(\S 2)$. We then demonstrate that in some circumstances, the proposed approach allows to exactly retrieve the strain profile in epitaxial films (§3). Finally, the method is applied to $\mathrm{SmNiO}_{3}$ films epitaxially grown on (001) $\mathrm{SrTiO}_{3}$ substrates by metal organic - chemical vapor deposition (§4).

\section{Diffraction from a film/substrate system}

In the following the diffraction from the film is treated within the framework of the kinematical theory of diffraction. This assumption is justified for thin films studied at nongrazing angles (Pietsch et al., 2004) and allows an easier handling of the expression of the diffracted amplitude than the dynamical theory. On the other hand, the diffraction from the substrate must be treated dynamically. If the film and substrate diffract coherently, then the total diffracted amplitude can be written (Holý et al., 1999) (see also Pietsch et al., 2004 ; Wie, 1994 ; Kyutt et al., 1980):

$$
E=E_{s}^{d y n} \exp (i q t)+i E_{f}
$$


where $E_{s}^{d y n}$ and $E_{f}$ are the amplitudes diffracted by the substrate (dynamically) and the film, respectively. $t$ is the film thickness and $q$ is the modulus of the reduced scattering vector, i.e. the deviation of the scattering vector $\mathbf{Q}$ from the Bragg position, including refraction and absorption corrections:

$$
q=|\mathbf{Q}-\mathbf{h}|+\frac{K x_{0}}{2 \gamma_{h}}\left(1-\frac{\gamma_{h}}{\gamma_{0}}\right)
$$

where $\chi_{0}$ is the polarizability, the real part of which accounts for refraction and the imaginary part accounts for absorption. $\mathbf{h}$ is the reciprocal lattice vector of the reflection considered with Bragg angle $\theta . \gamma_{0}$ and $\gamma_{\mathrm{h}}$ are the direction cosines of the incident and diffracted wave vectors ( $K=2 \pi / \lambda, \lambda$ being the wavelength) with respect to the inwards surface normal, i.e. $\gamma_{0}=\sin (\theta$ - $\phi$ ) and $\gamma_{h}=-\sin (\theta+\phi)$, where $\phi$ is the angle between the surface and the investigated lattice planes. Since we are interested in strain profiles along the surface normal, we shall only consider symmetrical reflections ( $\phi=0)$, so that the last term of equation (2) reduces to $K \chi_{0} /$ $\sin \theta$. The reduced scattering vector can also account for the effects of short range lattice spacing fluctuations which can be encountered in thin films. The modulus of the reduced scattering vector then reads (Boulle et al. 2006a; Boulle et al., 2006b)

$$
q=|\mathbf{Q}-\mathbf{h}|+\frac{K x_{0}}{\sin \theta}-i \frac{\sigma_{u}^{2} h^{2}}{2 d}
$$

where $d$ is the lattice spacing of the lattice planes considered and $\sigma_{\mathrm{u}}$ corresponds to the rootmean-squared (rms) lattice displacements. It is worth mentioning that an alternative formulation of the amplitude diffracted by a film/substrate system has been given by Zolotoyabko (1998):

$$
E=E_{s}^{k i n} \exp (i q t)+E_{f}
$$

where $E_{s}^{k i n}$ is the amplitude diffracted by the substrate using an advanced kinematical formulation and which perfectly reproduces all dynamical interference effects. Notice that in 
comparison with equation (1), the amplitude of the film here appears at $E_{f}$, and note $i E_{f}$. In the semi-kinematical expression (1) the exact dynamical formulation of the diffraction from the substrate is used, whereas in the kinematical expression (4) a kinematical formulation of the diffraction from the susbtrate is used. As pointed out by Zolotoyabko (1998), all attempts to use the exact dynamical formulation for the susbtrate's scattering amplitude in combination with equation (4) failed because such a 'mixture' does not correctly describe the phase relations between the wave scattered from the film and from the substrate.

Finally, if the film and the substrate diffract incoherently, the total diffracted intensity is given by $I=I_{s}+I_{f}(5)$, where $I_{s}$ and $I_{f}$ are the intensities diffracted by the the film and the substrate, respectively. The contributions of the film and the substrate to the total diffraction amplitude are detailed below.

\subsection{Contribution of the film}

Firstly we shall assume that the film does not exhibit compositional gradients, so that the structure factor can be considered constant throughout the film thickness. With this assumption, for a symmetrical reflection with vector $\mathbf{h}$, the amplitude diffracted by the film is

$$
E\left(q_{z}\right)=\frac{K X_{h}}{2 \sin \theta} \int_{0}^{\infty} d z \cdot \Omega(z ; t) \exp [i h u(z)] \exp \left(i q_{z} z\right)
$$

where $z$ is the coordinate along the surface normal, $\Omega(z ; t)$ is the shape factor of the film (i.e. $\Omega(z ; t)=1$ if $z \in[0, \mathrm{t}], \Omega(z ; t)=0$ otherwise $), u(z)$ is the displacement of the lattice from its ideal position at coordinate $z$, and $q_{z}$ is the $z$-component of the reduced scattering vector. $\chi_{h}$ is the $h$-th Fourier component of the polarizability which relates to the structure factor $F_{h}$ through $\chi_{h}=-r_{e} \lambda^{2} F_{h} / \pi V$ (where $r_{e}$ and $V$ are the classical electron radius and the unit cell volume) (Authier, 2005). 
Most thin films are not flat on the atomic scale and therefore exhibit a certain degree of thickness fluctuations. One must therefore consider the average amplitude

$$
\left\langle E\left(q_{z}\right)\right\rangle=\int d t \cdot p(t) E\left(q_{z}\right)
$$

where $p(t)$ is the thickness probability distribution function (PDF). Inserting equation (6) in equation (7) and changing the order of integration yields

$$
\left\langle E\left(q_{z}\right)\right\rangle=\frac{K X_{h}}{2 \sin \theta} \int_{0}^{\infty} d z \cdot\langle\Omega(z ; t)\rangle \exp [i h u(z)] \exp \left(i q_{z} z\right)
$$

and

$$
\langle\Omega(z ; t)\rangle=\int_{z}^{\infty} d t \cdot p(t) \Omega(z ; t)
$$

is the averaged shape factor. In deriving equation (8) we made the assumption that $u(z)$ is independent of the film thickness, i.e. we don't account for correlations between the strain profile and the film thickness. This assumption may be considered as justified for relatively small thickness fluctuations. The main interest of this assumption lies in the fact that equation (8b) can be analytically evaluated for several $p(t)$ which, hence, avoids the numerical evaluation of equation (7). Solution of $\langle\Omega(z ; t)>$ are here given for a normal PDF (notice that to avoid the occurrence of negative thicknesses, the normal PDF has to be constrained with condition $\sigma<\mu / 3$ (Boulle et al., 2006))

$$
p_{N}(t)=\frac{1}{\sqrt{2} \sigma} \exp \left[-\frac{1}{2}\left(\frac{t-\mu}{\sigma}\right)^{2}\right]
$$

a lognormal PDF

$$
p_{L N}(t)=\frac{1}{\sqrt{2 \pi} \sigma_{L N} t} \exp \left[-\frac{1}{2}\left(\frac{\ln t-\mu_{L N}}{\sigma_{L N}}\right)^{2}\right]
$$

and general histogram distribution consisting of $N$ classes, each thickness $t_{n}$ occurring with a probability $p_{n}$. In the above equations $\mu\left(\mu_{L N}\right)$ and $\sigma^{2}\left(\sigma_{L N}^{2}\right)$ are the mean (lognormal mean) 
and variance (lognormal variance) of the thickness PDF. The parameters $\mu_{L N}$ and $\sigma_{L N}{ }^{2}$ are related to their normal counterparts by $\mu=\exp \left(\mu_{L N}+\sigma_{L N}^{2} / 2\right)$ and $\sigma^{2}=\mu^{2}\left[\exp \left(\sigma_{L N}^{2}\right)-1\right]$. With these definitions equation ( $8 b$ ) becomes

$$
\langle\Omega(z ; t)\rangle_{N}=\frac{1}{2} \operatorname{erfc}\left(\frac{z-\mu}{\sqrt{2} \sigma}\right)
$$

for the normal PDF,

$$
\langle\Omega(z ; t)\rangle_{L N}=\frac{1}{2} \operatorname{erfc}\left(\frac{\ln z-\mu_{L N}}{\sqrt{2} \sigma_{L N}}\right)
$$

for the lognormal PDF and

$$
\langle\Omega(z ; t)\rangle_{H}=\sum_{n=1}^{N} p_{n} \Omega\left(z ; t_{n}\right)\left[t_{n}-\max \left(z, t_{n-1}\right)\right]
$$

for the histogram PDF. In general the thickness distribution is not known a priori, so that the parameters of the distribution ( $\mu$ and $\sigma$ ) are obtained by fitting the calculated profiles to experimental data. In this procedure the normal PDF is believed to be applicable in most cases $^{1}$. There are cases however, where the size distribution is known to obey a particular (non Gaussian) functional form. This is for instance the case for some nanoparticle systems which exhibit a lognormal size distribution (Kiss et al., 1999). Finally, if the thickness PDF is known from an independent measurement (such as atomic force microscopy observations for instance) the wisest choice is to use the histogram distribution obtained by this method.

In the following we restrict ourselves to the normal distribution. The effect of thickness fluctuations is illustrated in the inset figure 1: instead of exhibiting an abrupt variation, the shape factor smoothly decreases from 1 to 0 . The shape and width of the transition zone depends on the chosen PDF and the associated variance. Here the calculations were

1 Strictly speaking, since the thickness varies in a discrete fashion (i.e. by multiples of the lattice spacing) one should use discrete PDFs, for instance the binomial distribution instead of the normal distribution. In the present case we shall consider films with a thickness much greater than than the smallest height variation (i.e. much greater than the lattice spacing) so that continuous distribution can be safely used (Boulle et al., 2004, 2006a). 
performed for $50 \mathrm{~nm}$ - thick film exhibiting a normal PDF with $\sigma=0,5 \%$ of the film thickness $(2.5 \mathrm{~nm})$ and $10 \%$ of the film thickness $(5 \mathrm{~nm})$. The calculated diffraction curves are given in figure 1 for an unstrained film $(u(z)=0)$. The presence of thickness fluctuations has a significant influence on the diffraction curve as it results in a damping of the high order fringes: the higher the thickness fluctuations, the less the fringes are visible. In the case of very high thickness fluctuations the fringes can be completely smeared out (Boulle et al., 2005). We shall however not consider this case here as it is incompatible with the assumption detailed earlier, i.e. we restrict ourselves to small $\sigma$ s. Finally, the effect of roughness can be easily accounted for by multiplying equation (8a) by Debye-Waller - like factor, $\exp \left(-h^{2} \sigma_{r}^{2} / 2\right)$, where $\sigma_{r}^{2}$ is the rms roughness (Boulle et al., 2006a; Boulle et al., 2006b). This roughness term corresponds to height fluctuations at the film/substrate interface which are entirely replicated at the surface, i.e. it does not yield film thickness fluctuations.

\subsection{Contribution of the substrate}

In the framework of equation (1) the expression of the amplitude diffracted by the substrate is given by the dynamical theory of diffraction and can be found for instance in Authier (2005). In the framework of equation (4) Zolotoyabko (1998) derived the following equation

$$
E_{s}^{k i n}=\frac{K X_{h}}{2 \sin \theta} \frac{d}{1-\left(1-d / \Lambda_{e}\right) \exp (i Q d)}
$$

where $\Lambda_{e}$ is the penetration depth due to extinction, $\Lambda_{e}=\lambda \sin \theta / \pi\left|\chi_{\mathrm{n}}\right|$. The effect of absorption is included in the scattering vector $Q$. We here perform the same derivation so as to express the diffracted amplitude in terms of $q_{z}$ instead of Q. We obtain

$$
E_{s}^{k i n}=\frac{K X_{h}}{2 \sin \theta} \frac{1}{1 / \Lambda_{e}-i q_{z}}
$$

We show below that this last expression, when used in equation (4), yields exactly the same 
results as a full dynamical calculation. Strictly speaking, the only part of the diffraction curve that is not perfectly reproduced is the total reflection domain of the substrate peak. However, as pointed out by Zolotoyabko (1998) this detail is unimportant because in practice this narrow region ( \pm 1 Darwin width) is broadened by the finite resolution of the diffractometer.

\subsection{The total diffraction profile}

In the following all calculations are performed assuming $50 \mathrm{~nm}$ - thick $\mathrm{SmNiO}_{3}$ films deposited on (001) $\mathrm{SrTiO}_{3}$ substrates. $\mathrm{SrTiO}_{3}$ is a cubic perovskite (space group $\mathrm{Pm}-3 \mathrm{~m}$ ) with lattice parameter $\mathrm{a}=3.905 \AA . \mathrm{SmNiO}_{3}$ is an orthorhombically distorted perovskite (space group Pbmn, $\mathrm{a}=5.328 \AA, \mathrm{b}=5.437 \AA, \mathrm{c}=7.568 \AA$ ) which can be described in a pseudocubic unit cell with $\mathrm{a}=3.798 \AA$. Previous studies on this material (Conchon et al., 2007a; Conchon et al., 2007b) revealed that the lattice parameter is higher than expected, i.e. we have $\mathrm{a}=3.844 \AA$ (the origin of this discrepancy is discussed in $\S 4$ ). The associated misfit strain is $1.6 \%$.

The calculated diffraction curve using these parameters is displayed in figure $2 \mathrm{a}$ for the $(002)$ reflection. The black curve has been calculated assuming that both the film and the substrate diffract dynamically for a film perfectly lattice-matched on the substrate. The red curve labeled (1) has been calculated using equation (4), together with equations (8), (9) and (10) without thickness fluctuations $(\sigma=0)$. It can be seen that the calculation using equation (4) perfectly matches the dynamical calculation. Besides, if the film and the substrate are assumed to diffract incoherently, i.e. the total intensity is the sum of the intensities diffracted by the film and the substrate, the diffraction exhibit a strongly modified fringes structure. This situation is illustrated in figure $2 \mathrm{~b}$ with the curve labeled (1) (the black curve representing the dynamical calculation is also shown for each calculated curve for comparison purposes). 
Let us now consider the effects of thickness fluctuations. In the latter case (incoherent film/substrate diffraction, figure $2 b$ ), the fringes structure is affected in the same way as in the case of the diffraction from an isolated film (section 1, figure 1), i.e. the fringes are damped. This behavior is easily understood since there are no interferences between the waves diffracted by the film and the substrate. Conversely, in the case of a coherent film/substrate diffraction (figure 2a), the interferences between the waves diffracted by the film and the substrate give rise to significant modification of the fringes structure when thickness fluctuations are considered (curves (2) and (3), figure 2a).

In the light of this analysis, it appears that the diffraction curve is highly sensitive on the presence thickness fluctuations and on the presence of a coherently diffracting substrate. Since strain profiles also affect the fringes structure (Boulle et al., 2003) one must be able to distinguish between strain - induced effects and thickness fluctuations - induced effects. This can be achieved by analyzing several orders of reflections from the same crystallographic planes family, e.g. several (00l) reflections. Indeed, according to equation (8), strain effects scale with reciprocal lattice vector $\mathbf{h}$, whereas effects related to thickness fluctuations affect all reflections identically. On the other hand, there is no a priori mean to detect whether the waves diffracted by the film and by the substrate couple coherently or not. Both hypothesis have therefore to be tested. However, it is more likely in partially relaxed structures (and a fortiori in strongly relaxed) that the coherency between the film and the substrate is lost because of misfit dislocations lying at the interface (Fewster, 1992). Finally, it is worth noticing that, in a similar fashion to what is done with other methods (the laminae method in combination with the Takagi-Taupin equations, for instance), since we here only consider the coherent part of the diffracted intensity, the diffuse scattering emanating from defects is neglected. 


\section{The strain profile}

In the following we decompose the displacement profile into third degree B-spline basis functions:

$$
u(z)=\sum_{i=1}^{N} w_{i} B_{i, 3}(z)
$$

where $w_{i}$ is the weight of the $\mathrm{i}$-th B-spline of third degree, $B_{i, 3}(z)$ and $N$ is the number of knots chosen to compute $u(z)$ (Boulle et al., 2003). In writing the above equation, the displacement is implicitly assumed to be correctly described by a cubic spline function. The advantages of this assumption are twofold. Firstly, the cubic spline has two continuous derivatives which hence avoids abrupt variations. Secondly, for a given number of knots, the cubic spline interpolates with a minimum curvature which hence avoids unphysical oscillations that can be encountered in the inversion of experimental data. The obvious drawback of the present approach is that it is not suited to systems where abrupt variations of $u(z)$ are indeed expected, as in the case of multilayers for instance where the lattice parameter (and hence $u(z)$ ) changes abruptly at each interface. In such a case another approach must be used (Dilanian et al., 2006).

Another interesting feature of cubic B-spline functions lies in their high versatility: the degree of details of the displacement profile that can be rendered entirely depends on the number of knots. Increasing the number of knots increases the ability to render tiny features of $u(z)$, but at the same time it increases the possibility for $u(z)$ to exhibit wild oscillations. On the contrary, decreasing the number of knots induces a smoothing of $u(z)$ but increases the possibility to wipe-out small details. In practice we have found that around ten knots appears as a good compromise. An example of displacement profile (red curve) and the associated basis functions (black curves) are depicted in figure 3 (this curve corresponds to the strain 
profile (3) in figure 4a discussed in the next section). In this case the film is divided into 9 intervals and 13 knots are required to describe the displacement profile over the whole film thickness. As mentioned in the introduction, least square fitting - based methods often rely on the choice of a particular guess model for $u(z)$ (either a physically sound or arbitrarily chosen model). The method presented here is an alternative to these methods when there is no initial guess for $u(z)$, i.e. when the nature of the defect responsible for the strain profile is unknown.

\subsection{Strain profile retrieval}

In this section we shall test the ability of the method to actually retrieve the strain profile. We choose the same conditions as in $\S 2.3\left(\mathrm{SmNiO}_{3} / \mathrm{SrTiO}_{3}, \mathrm{t}=50 \mathrm{~nm}\right)$. We further assume that the waves diffracted by the film and the substrate couple coherently and we assume perfectly flat films (no thickness fluctuations). These assumptions have no consequences on the conclusions drawn in this section. Diffraction curves have been calculated using three different input $u(z)$ functions. The strain profiles corresponding to these three different $u(z)$ profiles are shown as symbols in figure 4a. The left axis indicates the strain, whereas the right axis indicates the degree of strain relaxation:

$$
R(z)=\frac{a_{/ /}(z)-a_{s}}{a_{f}-a_{s}}
$$

where $a_{/ /}(z)$ is the in-plane lattice parameter at the coordinate $z, a_{f}$ and $a_{s}$ are the strain free lattice parameters of the film and the substrate, respectively. $R(z)=0$ for a perfect lattice matching, whereas $R(z)=100 \%$ for a relaxed film (Pietsch et al., 2004). The strain profile labeled (1) correspond to a smooth strain relaxation across the film thickness. The strain profiles (2) and (3) exhibit a strong strain relaxation close to the interface and, for the profile (3), surface lattice expansion. The corresponding diffraction curves are shown as thick black lines in figure $4 \mathrm{~b}$. It can be readily observed that the strain profile has profound incidence of 
the diffraction curve, and especially on the fringes structure.

These curves have been used as "data" and the model has been fitted to these data. Starting from a displacement equal to $0(u(z)=0)$, the parameters entering the model (i.e. $t$ and the different B-spline weights) are optimized by minimizing the criterion (Klappe \& Fewster, 1994; Milita \& Servidori, 1995)

$$
\operatorname{Err}=\sum_{i=1}^{M}\left[\log \left(I_{i}^{f i t}\right)-\log \left(I_{i}^{o b s}\right)\right]^{2}
$$

where $M$ is the number of data points and $I_{i}^{\text {fit }}$ and $I_{i}^{\text {obs }}$ are the fitted and observed intensities. The parameters have been refined using a Levenberg-Marquardt minimization algorithm (Press et al., 2002). The fitted intensities are drawn as thin red lines in figure $4 \mathrm{~b}$ : the fitted curve matches the observed curve exactly. The retrieved strain profiles are drawn as full lines in figure 4a. These profiles perfectly reproduce the shape of the input strain profile (symbols). It can be concluded that the approach presented here allows to retrieve strain profiles in epitaxial films from the X-ray diffraction profile even without an initial guess model, provided however that the actual strain profile can be described by cubic spline function. It can be expected that in an actual experiment the presence of experimental noise will increase the number of possible solutions produced by the least-square fitting procedure (Provencher, 1982). The use of cubic spline functions is here of particular interest since it implicitly puts smoothness and curvature constraints on the possible solutions, thereby limiting the occurrence of unphysical oscillating solutions.

\section{Application}

The method exposed above has been applied to the determination of strain profiles in $\mathrm{SmNiO}_{3}$ films grown on (001) $\mathrm{SrTiO}_{3}$ substrates by metal organic - chemical vapor deposition (Conchon et al., 2007a). In order to achieve the highest dynamic range and hence to maximize 
the fringes contrast, the experiments were carried out at the BM2 beamline at the European Synchrotron Radiation Facility (ESRF, Grenoble, France). The beam energy was set to 20 $\mathrm{keV}$. The incident beam is monochromated by a (111) Si two-crystal monochromator. A (111) Si crystal has been used as an analyzing crystal (Ferrer et al., 1998). The (002) and (004) reflections have been recorded and analyzed (only the (002) is shown here). In order to account for the finite resolution of the instrument, the calculated curve has been convoluted with Gaussian function whose width has been fixed to the value of the peak width of the substrate reflection. Finally a constant background has been added to the calculated curve in order to account for the actual background level.

Three different models have been fitted to the data. The first one assumes a coherent film/substrate diffraction and neglects thickness fluctuations. This case correspond to the usual model and is valid for perfect structures. The second model includes thickness fluctuations (we here used the normal PDF). The third model assumes incoherent film/substrate diffraction and also includes thickness fluctuations. The three simulations, (labeled from 1 to 3) are displayed in figure 5. Enlarged views of the left and right tails of the peaks are given in the inset (a) and (b). All three models fit the central part of the peak fairly well, the main differences are visible in the profile tails. Model (1) clearly fails to reproduce the fringe structure, in particular the fringes are out of phase on the left hand side. The addition of thickness fluctuations slightly improves the agreement on the right hand side (although the fringes are slightly dephased) but the simulation is clearly not acceptable. The best agreement is obtained with the last model. The fringes structure is fairly well reproduced over the whole angular range. The obtained film thickness is $84 \mathrm{~nm}$ and the rms thickness fluctuation is $1 \mathrm{~nm}$. In the present case the loss of coherency between the waves diffracted by the film and the substrate can be easily understood. The analysis of asymmetrical reciprocal 
space maps indeed revealed that the film exhibit a 7\% strain relaxation (Conchon et al., 2007a, 2008b). It can hence be expected that the strain fields induced by the misfit dislocations lying at the interface disrupt the wavefield in the film/substrate strucure leading to an incoherent coupling of the waves diffracted by the film and the substrate (Fewster, 1992).

The strain profiles obtained from the simulation procedure are displayed in figure 6 . The strain profiles exhibit marked differences, especially close to the interface where model (2) and (3) predict an abrupt decrease of the strain from $1.5 \%$ to 0 in the 5 first nanometers. This behaviour is not predicted by model (1) which is the only one that does not account for thickness fluctuations. The inclusion of film thickness fluctuations in the model hence appears as a critical parameter to retrieve the strain profile. For all models the strain oscillates between $0.5 \%$ and $-0.5 \%$ with a similar shape for three models.

At first sight the high tensile strain close to the interface seems to be paradoxical. Indeed, since the film is tensily strained in the interface plane, one would expect the film to be compressively strained in the vertical direction. A detailed inspection of the $\mathrm{SmNiO}_{3} / \mathrm{SrTiO}_{3}$ system (Conchon et al., 2007c) revealed that the in-plane strain destabilizes the $\mathrm{Ni}^{3+}$, which then transforms into $\mathrm{Ni}^{2+}$ (this is accompanied by the formation of oxygen vacancies for charge conservation). $\mathrm{Ni}^{2+}$ having a larger ionic radius, the $\mathrm{SmNiO}_{3}$ unit cell volume increases upon the $\mathrm{Ni}^{3+} \rightarrow \mathrm{Ni}^{2+}$ transformation. This is why we observe a dilatation in the vertical direction instead of the expected contraction (Conchon et al., 2007c). The strain profile hence suggests that the oxygen vacancies are mainly located at the interface (between 0 and $5 \mathrm{~nm}$ ). This can be understood considering that this is the region where the influence of the interfacial strain is maximum. Between 5 and $20 \mathrm{~nm}$ the strain is compressive indicating that there are no oxygen vacancies, i.e. this compressive strain is the response to the in-plane 
tensile strain. The following oscillations are somewhat fussy to interpret. However, since the three profiles have similar shape these oscillations can hardly be attributed to random errors in the simulation procedure. Since the oxygen content is critical in the formation of oxygen vacancies and hence on the $\mathrm{Ni}^{3+} \rightarrow \mathrm{Ni}^{2+}$ transformation, the observed oscillations could be due to instabilities of oxygen pressure during deposition.

\section{Conclusions}

We have presented a least-square fitting - based method that allows to retrieve strain profiles in epitaxial films using XRD. The method is model-independent in the sense that it does not require any guess model for the shape of the strain profile. For that purpose the displacement profile across the film thickness is modeled using cubic B-spline functions. These functions are extremely versatile since they can adopt almost any shape while avoiding abrupt discontinuities and wild oscillations. The coherent or incoherent diffraction from the substrate is easily taken into account. In the case of a coherent contribution from the substrate, the present model yields the same results as the dynamical theory. Thickness fluctuations are explicitly included in the model and have been described with a normal, a lognormal and a histogram thickness probability distribution function. These thickness fluctuations were found to have a profound influence on the diffraction profile. Provided that the actual strain profile can be described by a cubic spline function, it has been shown from simulations that the present model allows to exactly retrieve the strain profile in epitaxial films. Finally, the model has been applied to the determination of strain profiles in $\mathrm{SmNiO}_{3}$ films epitaxially grown on (001) $\mathrm{SrTiO}_{3}$. In this case it has been found that the film/substrate coherence is broken by the presence of misfit dislocations and the shape of the retrieved strain profile has been discussed in terms of oxygen vacancies. 


\section{Acknowledgments}

We are grateful to the BM2 beamline staff (J. F. Bérar, N. Boudet, E. Dooryhée, J. L. Hodeau) for assistance during the experiments. F. C. expresses her gratitude towards the FAME European Network of Excellence for financial support. Finally we would like to thank C. Girardot and S. Pignard for providing the $\mathrm{SmNiO}_{3} / \mathrm{SrTiO}_{3}$ samples.

\section{References}

Authier, A. (2005) Dynamical theory of X-ray diffraction, IUCr monographs on crystallography, 11. Oxford University Press, reprinted with revisions.

Bartels, W. J., Hornstra., J., Lobeek, D. J. W. (1986) Acta. Cryst. A 42, 539-545.

Boulle, A., Masson, O., Guinebretière, R., Dauger, A. (2003) J. Appl. Cryst. 36, 1424-1431.

Boulle, A., Guinebretière, R., Dauger, A. (2004) J. Phys. IV (France) 118, 183-191.

Boulle, A., Conchon, F., Guinebretière, R. (2006) Acta Cryst. A 62, 11-20.

Boulle, A., Guinebretière, R., Dauger, A. (2006a) Z. Kristallogr. Suppl. 23, 123-128.

Boulle, A., Guinebretière, R., Masson, O., Bachelet, R., Conchon, F., Dauger, A. (2006b) Appl. Surf. Sci. 253, 95-105.

Catalan, G., Noheda, B., McAneney, J., Sinnamon, L. J., Gregg, J. M. (2005) Phys. Rev. B 72, 020102(R)-1-4.

Conchon, F., Boulle, A., Girardot, C., Pignard, S., Guinebretière, R., Dooryhée, E., Hodeau, J. L., Weiss, F., Kreisel, J., Bérar, J. F. (2007a) J. Phys. D: Appl. Phys. 40, 4872-4876.

Conchon, F., Boulle, A., Girardot, C., Pignard, S., Guinebretière, R., Dooryhée, E., Hodeau, J. L., Weiss, F., Kreisel, J. (2007b) Mat. Sci. Eng. B 144, 32-37.

Conchon, F., Boulle, A., Guinebretière, R., Girardot, C., Pignard, S., Kreisel, J., Weiss, F., 
Dooryhée, E., Hodeau, J. L. (2007c) Appl. Phys. Lett. 91, 192110-1-3.

Conchon, F., Boulle, A., Guinebretière, R., Girardot, C., Pignard, S., Kreisel, J., Weiss, F., Dooryhée, E., Hodeau, J. L. (2008a) J. Phys.: Condens. Matter, 145216-1-7.

Conchon, F., Boulle, A., Guinebretière, R., Dooryhée, E., Hodeau, J. L., Girardot, C., Pignard, S., Kreisel, J., Weiss, F., Libralesso, L., Lee, T. L. (2008b) J. Appl. Phys 103, 123501-1-7.

Diaz, B., Abramof, E., Castro, R. M., Ueda, M., Reuther, H. (2007) J. Appl. Phys. 101, 103523-1-6.

Dilanian, R. A., Nikulin, A. Y., Darahanau, A. V., Hester, J., Zaumseil, P. (2006) J. App. Phys. 99, $113526-1-5$.

Ferrer, J. L., Simon, J. P., Bérar, J. F., Caillot, B., Fanchon, E., Kaïkati, O., Arnaud, S., Guidotti, M., Pirocchi, M., Roth, M. (1998) J. Synchrotron Rad. 5, 1346-1356.

Fewster, P. F. (1992) J. Appl. Cryst. 25, 714-723.

Halliwell, M. A. G., Lyons. M. H., Hill, M. J. (1984) J. Cryst. Growth 68, 523-531.

Hironaka, Y., Yazaki, A., Saito, F., Nakamura, K. G., Takenaka, H., Yoshida, M. (2000) Appl. Phys. Lett. 77, 1967-1969.

Holý, V., Pietsch, U., Baumbach, T. (1999) High resolution X-ray scattering from thin films and multilayers, New-York: Springer-Verlag.

Kiss, L. B., Soderlund, J., Niklasson, G. A., Granqvist, C. G. (1999) Nanotechnology 10, $25-28$.

Klappe, J. G. E., Fewster, P. F. (1994) J. Appl. Cryst. 27, 103-110.

Kyutt, R. N., Petrashen, P. V., Sorokin, L. M. (1980) Phys. Stat. Sol. (a) 60, 381-389.

Leclerc, S., Declémy, A., Beaufort, M. F., Tromas, C., Barbot, J. F. (2005) J. Appl. Phys. 98, 113506-1-6. 
Milita, S., Servidori, M. (1995), J. Appl. Cryst. 28, 666-672.

Nicola, L., Van der Giessen, E., Gurtin, M. E. (2005) J. Mech. Phys. Sol. 53, 1280-1294.

Nikulin, A. Y. (1998) Recent Res. Devel. Applied. Phys. 1, 1-21.

Pietsch, U., Holý, V., Baumbach, T. (2004) High resolution X-ray scattering - from thin films to lateral nanostructures, New-York: Springer-Verlag.

Press, W. H., Teukolsky, S. A., Vetterling, W. T., Flannery, B. P. (2002) Numerical Recipes in C, Cambridge University Press (http://www.nr.com/).

Provencher, W. (1982). Comp. Phys. Com. 27, 229-242.

Siegle H., Hoffmann, A., Eckey, L., Thomsen, C., Christen, J., Bertram, F., Schmidt, D., Rudloff, D., Hiramatsu, K. (1997) Appl. Phys. Lett. 71, 2490-2492.

Shen, Q., Kycia, S. (1997) Phys. Rev. B 55, 15791-15797.

Sousbie, N., Capello, L., Eymery, J., Rieutord, F., Lagahe, C. (2006) J. Appl. Phys. 99, 103509-1-7.

Steinfort, A. J., Scholte, P. M. L. O., Ettema, A., Tuinstra, F., Nielsen, M., Landemark, E., Smilgies, D. M. (1996). Phys. Rev. Lett. 77, 2009-2012.

Takagi, S. (1969) J. Phys. Soc. Jpn 26, 1239-1253.

Taupin, D. (1964) Bull. Soc. Franç. Minér. Crist. 87, 469-511.

Vartanyants, I., Ern, C., Donner, W., Dosch, H., Caliebe, W. (2000) Appl. Phys. Lett. 77, 3929-3931.

van der Veen, F., Pfeiffer, F. (2004) J. Phys.: Condens. Matter 16, 5003-5030.

Wie, C. R. (1994) Mater. Sci. Eng. R 13, 1-56.

Wierzchowski, W., Wieteska, K., Graeff, W., Turos, A., Grötzschel, R. (2005) Vacuum 78, 569-575.

Zaumseil, P., Winter, U., Cembali, F., Servidori, M., Sourek, Z. (1987) Phys. Stat. Sol. (a) 
100, 95-104.

Zolotoyabko, E. (1998) J. Appl. Cryst. 31, 241-251. 


\section{Figure caption}

Figure 1: influence of thickness fluctuations on the diffraction curve of a $50 \mathrm{~nm}$ - thick film. (1): $\sigma=0$ (black curve); (2): $\sigma=5 \%$ of the film thickness (2.5 nm) (blue curve); (3): $\sigma=10$ $\%$ of the film thickness $(5 \mathrm{~nm})$ (red curve). Increasing thickness fluctuations results in a damping of the fringes. The curves are shifted vertically for clarity. Inset: corresponding averaged shape factors. For $\sigma>0$ the shape factor decreases smoothly from 1 to 0 .

Figure 2: influence of thickness fluctuations on the diffraction curve of a film/substrate system with film thickness $t=50 \mathrm{~nm}$ (thin red curves). (a): the film and substrate diffract coherently. (b): the film and substrate diffract incoherently. In each figure, (1): $\sigma=0 ;(2): \sigma=$ $5 \%$ of the film thickness $(2.5 \mathrm{~nm}) ;(3): \sigma=10 \%$ of the film thickness $(5 \mathrm{~nm})$. The thick black curve corresponds to the dynamical calculation. The curves are shifted vertically for clarity.

Figure 3: example plot the cubic B-spline basis functions (black curves) and the associated displacement profile (red curve). The displacement profile is a cubic spline function.

Figure 4: (a): input (symbols) and retrieved (lines) strain profiles for a $50 \mathrm{~nm}$ - thick film. The retrieved curves exactly reproduce the input curves. (b): diffraction curves corresponding to each of the strain profiles (labeled (1), (2) and (3)). The thick black curves are the diffraction profiles calculated using the input strain profiles. The thin red lines correspond to the least-square fitted curves. The fitted curves match the calculated curves perfectly. 
Figure 5: (002) diffraction curve of $\mathrm{SmNiO}_{3}$ epitaxially grown on $\mathrm{SrTiO}_{3}$. The curves labeled from (1) to (3) correspond to simulations performed with models (1), (2) and (3), respectively (see text for details). Black curve: experimental data; red curve: fitted curve. The best fit is achieved with model (3). The curves are shifted vertically for clarity. Inset (a): enlarged view of the left tail. Inset (b): enlarged view of the right tail.

Figure 6: experimental strain profiles retrieved with model (1) (squares, black line), model (2) (circles, blue line) and model (3) (triangles, red line). Inset: enlarged view of the 0-20 nm region. 
Figure 1

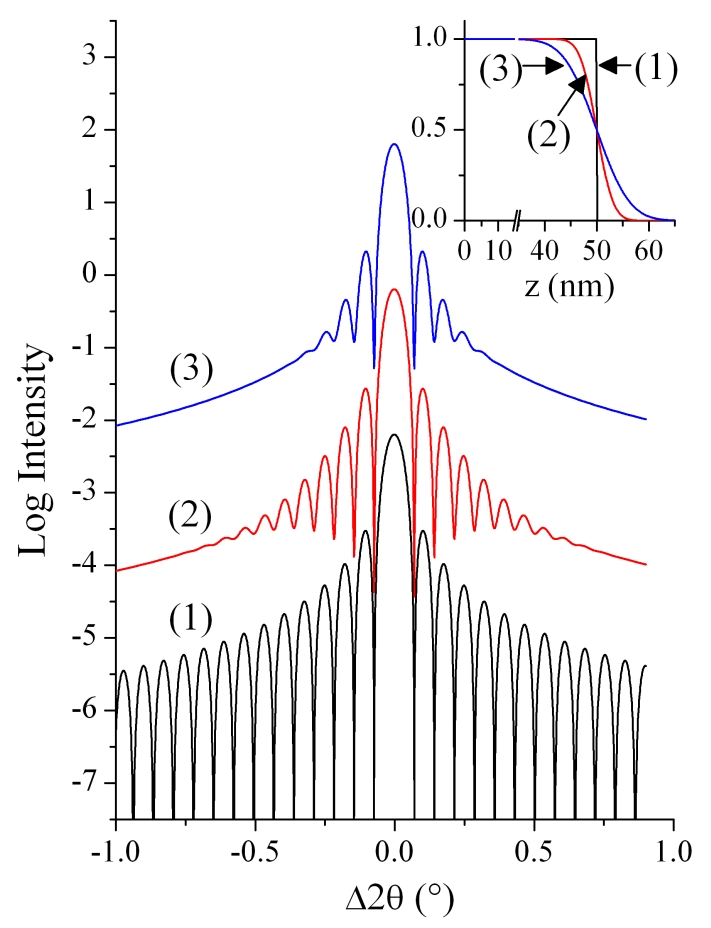


Figure 2
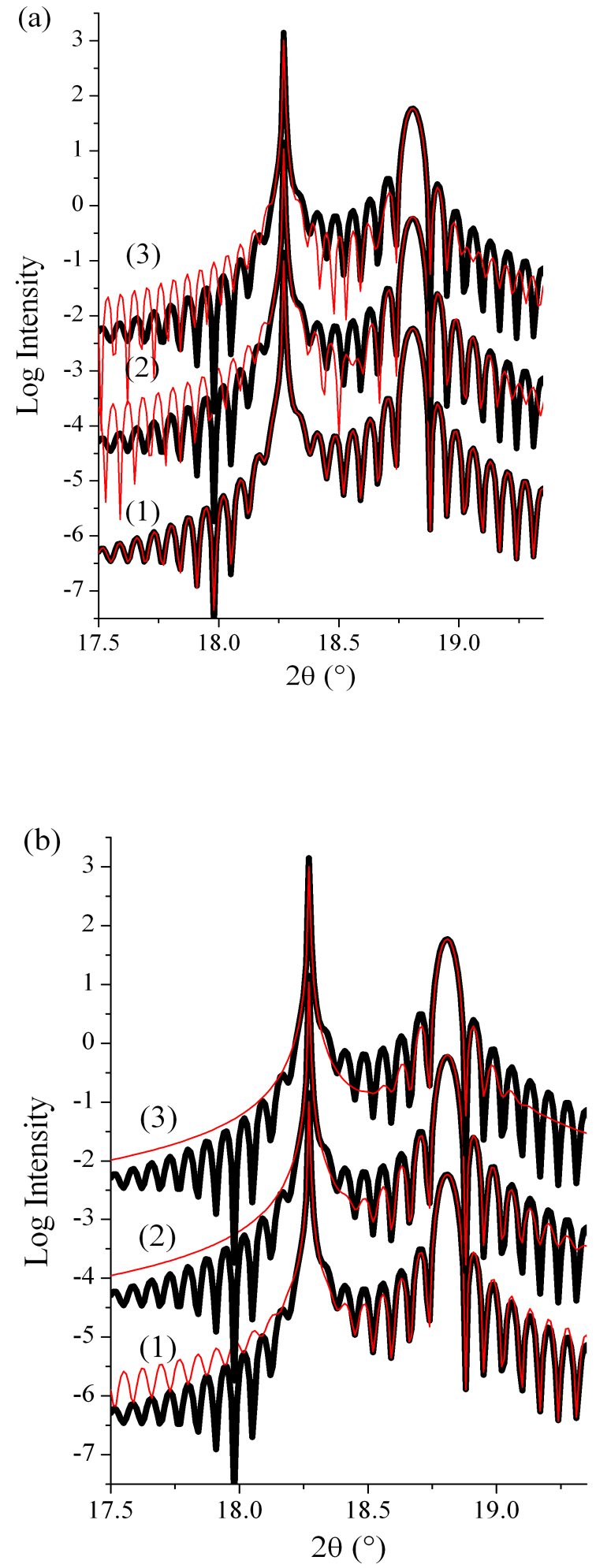
Figure 3

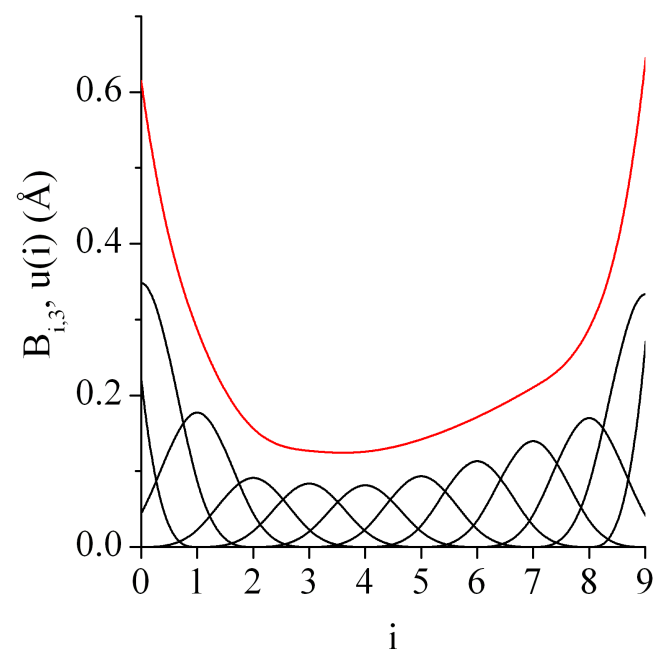


Figure 4
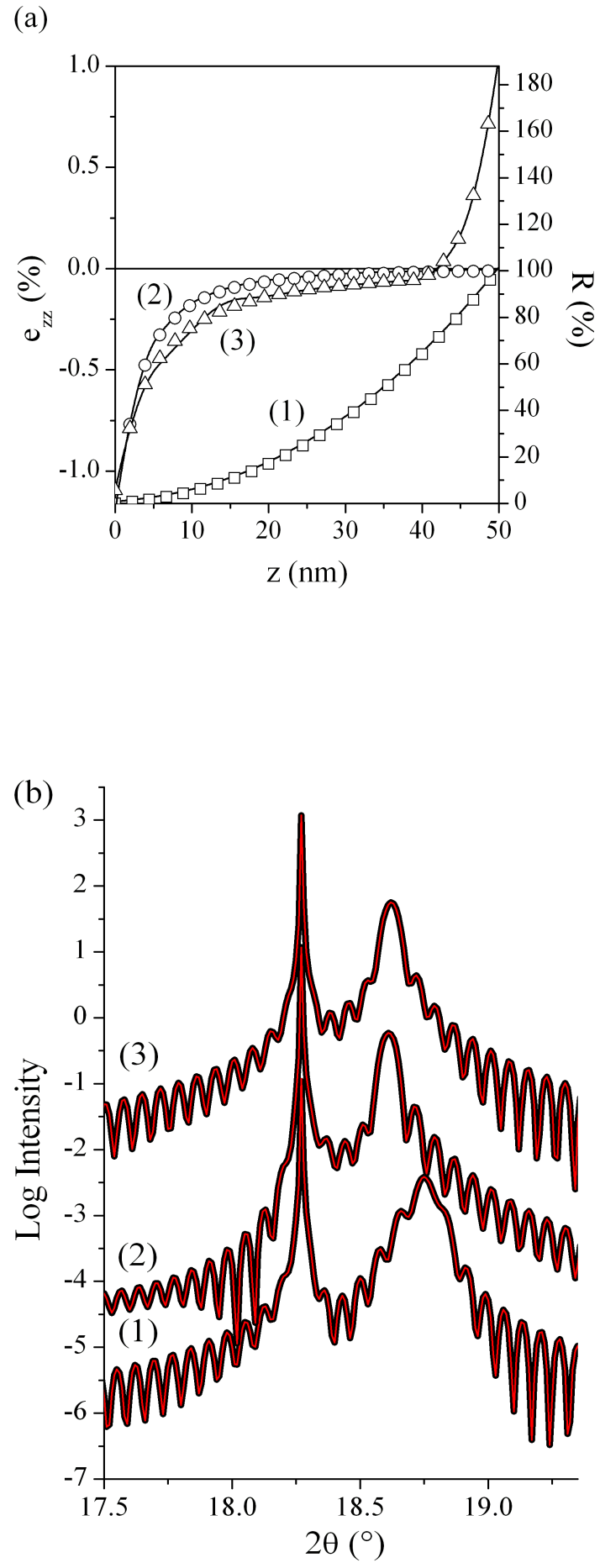
Figure 5
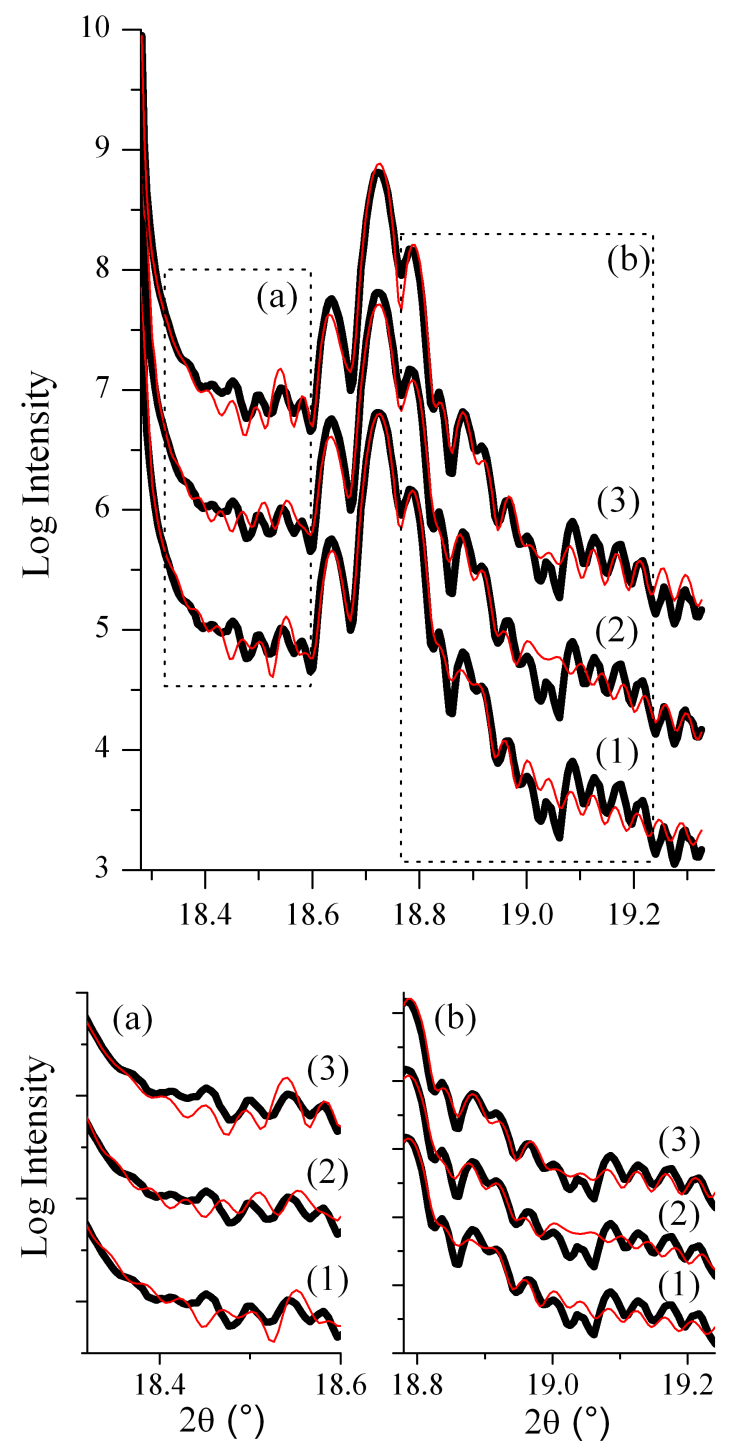
Figure 6

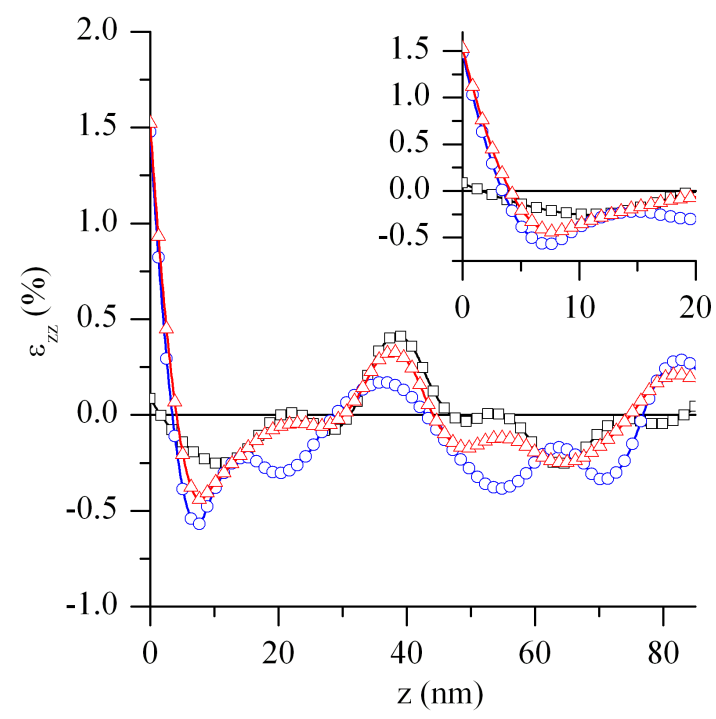

